\title{
e-Migrinter
}

$14 \mid 2016$

L'ajustement méthodologique comme fabrique critique du savoir dans les études migratoires

Bessora ; Barroux, Alpha, Abidjan-Gare du Nord

\section{Sarah Przybyl}

\section{(2) OpenEdition}

Journals

Édition électronique

URL : https://journals.openedition.org/e-migrinter/719

DOI : 10.4000/e-migrinter.719

ISSN : 1961-9685

Éditeur

UMR 7301 - Migrinter

\section{Référence électronique}

Sarah Przybyl, «Bessora ; Barroux, Alpha, Abidjan-Gare du Nord », e-Migrinter [En ligne], 14 | 2016, mis en ligne le , consulté le 20 mai 2021. URL : http://journals.openedition.org/e-migrinter/719; DOI : https:// doi.org/10.4000/e-migrinter.719

Ce document a été généré automatiquement le 20 mai 2021.

Tous droits réservés 


\title{
Bessora ; Barroux, Alpha, Abidjan- Gare du Nord
}

\author{
Sarah Przybyl
}

\section{RÉFÉRENCE}

Bessora (auteur) ; Barroux (illustrateur) (2014) Alpha, Abidjan-Gare du Nord, Paris, Gallimard, $126 \mathrm{p}$.

1 Les supports pour comprendre et analyser les migrations internationales se diversifient. Si le laboratoire MIGRINTER a fait du web un nouvel espace de valorisation de la recherche (photothèque, archives numérisées, réseaux sociaux, etc.), les rayons de son fonds documentaire ne cessent de s'étoffer et de se diversifier. Aux côtés des revues spécialisées, des atlas, des dictionnaires, une étagère accueille désormais des bandes dessinées. Ainsi, on retrouvera une adaptation du célèbre roman l'Étranger d'Albert Camus (Camus, Fernandez, 2013), ou encore l'histoire dessinée d'un "Beurgeois » (Boudjellal, 1997). Supports étonnants à première vue, ces ouvrages illustrés ont pourtant tous bel et bien un lien avec la thématique des migrations internationales. Ils permettent de lire et d'aborder ce sujet de manière différente. 
Illustration $n^{\circ} 1$ : Couverture de l'ouvrage Alpha, Abidjan-Gare du Nord.

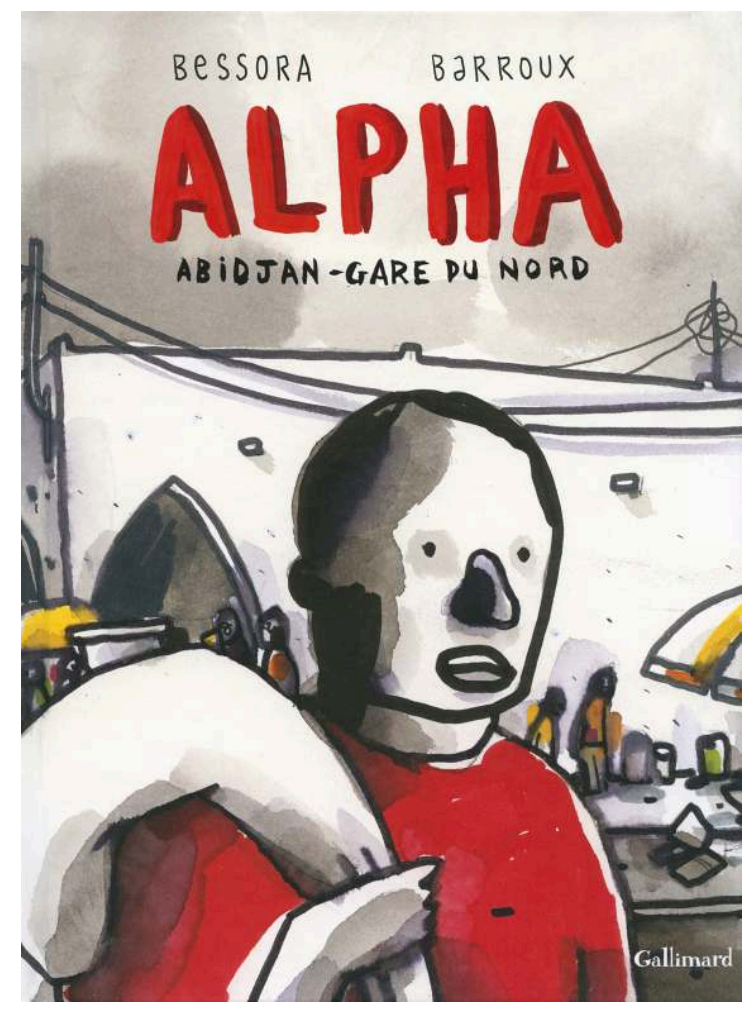

Extrait de : Bessora (auteur) ; Barroux (illustrateur) (2014) Alpha, Abidjan-Gare du Nord, Paris, Gallimard.

2 Choisir une bande dessinée c'est prendre le temps d'apprécier la signature artistique du dessinateur. Celle de Barroux interpelle. La simplicité du trait de l'illustrateur est mise au service d'une sobriété qui, au-delà de la couverture de l'ouvrage, se retrouvera tout au long du récit. Les teintes sombres majoritairement déployées dans ce livre laissent place à des sursauts colorés. Les dessins de Barroux sont irréguliers, ils percutent le regard. Le jeu de couleurs singulier et l'imprécision des traits épousent à la perfection les contours du parcours migratoire d'Alpha. C'est avec lui que le lecteur embarquera pour un long voyage, d'Abidjan (Côte d'Ivoire) jusqu'à la Gare du Nord (Paris).

Plus que l'histoire de la migration d'un homme, le récit du périple d'Alpha résonne avec les parcours et expériences de nombreux autres migrants qui prennent la route au péril de leur vie pour rejoindre l'Europe. Après s'être endetté pour financer le voyage de sa femme et de son fils en France, Alpha décide de les rejoindre et de quitter à son tour la Côte d'Ivoire. Ce départ apparait tant comme le signe d'un avenir meilleur que comme le sacrifice d'une vie. 
Illustration $n^{\circ} 2$ : Le jour du départ d'Alpha.

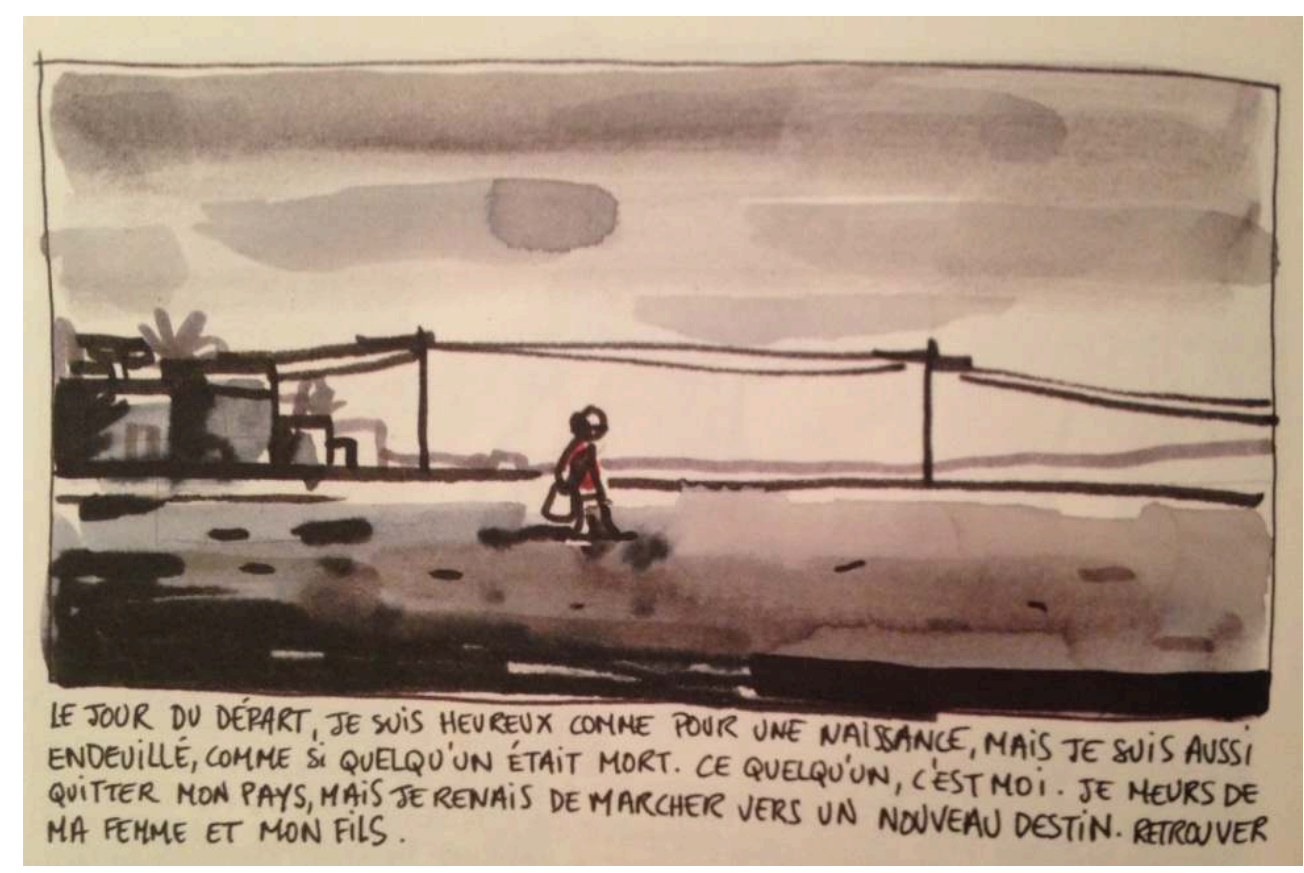

Extrait de : Bessora (auteur) ; Barroux (illustrateur) (2014) Alpha, Abidjan-Gare du Nord, Paris, Gallimard.

4 C'est avec un chauffeur de taxi qu'Alpha choisit de rejoindre le Mali par la route. Son parcours migratoire le conduira à faire la rencontre de nouveaux personnages qui participeront d'une manière ou d'une autre au voyage d'Alpha. Ces différents " aventuriers » dévoilent les nombreux acteurs qui dessinent aujourd'hui les contours des flux migratoires vers la France. Des passeurs, des femmes, des trafiquants, des enfants, des hommes se côtoient dans cette histoire et jalonnent les différentes étapes $\mathrm{du}$ parcours d'Alpha. Venus de différents pays d'Afrique subsaharienne, ces personnages donnent à voir le visage contemporain des migrations internationales. Sur les routes, Alpha rencontrera également des associations de soutien aux migrants auprès desquelles il trouvera réconfort et soutien. Enfin, si les décideurs politiques en matière d'immigration ne sont pas physiquement incarnés au cours de cette histoire, ils sont néanmoins bien présents. Les obstacles administratifs, les murs ou encore les contrôles que devront passer Alpha et ses compagnons de route matérialisent une présence à distance de ces acteurs incontournables.

La rudesse du parcours d'Alpha et sa détermination à continuer décuplent la force de ces rencontres. Dans le désert du Sahara, entre la chaleur étouffante et l'extrême froideur de la nuit, dans les remous de la mer Méditerranée, le texte de Bessora nous invite à partager une histoire, mais surtout une expérience migratoire sur les routes de l'ailleurs.

6 L'histoire d'Alpha c'est celle d'un homme animé par le désir de retrouver sa femme et son fils. Le propos léger et parfois humoristique de Bessora rappelle que les migrations internationales sont avant tout des histoires humaines. Si les routes sont bien incertaines, la détermination de ces « aventuriers $»^{1}$ reste intacte au cours de la lecture. Cette bande dessinée ce n'est pas seulement l'histoire d'Alpha que Bessora et Barroux donnent à voir et à lire; c'est aussi celle de milliers d'autres hommes, de femmes et d'enfants prêts à tous les sacrifices pour rejoindre un ailleurs plus prometteur. La seule 
inconnue du voyage reste le sort que cet ailleurs leurs réservera une fois arrivé à destination, mais aussi et surtout : Alpha retrouvera-t-il enfin sa femme et son fils pour lesquels il a pris tant de risques?

\section{Illustration $n^{\circ} 3$ : D'Abidjan à Paris, le parcours migratoire d'Alpha.}

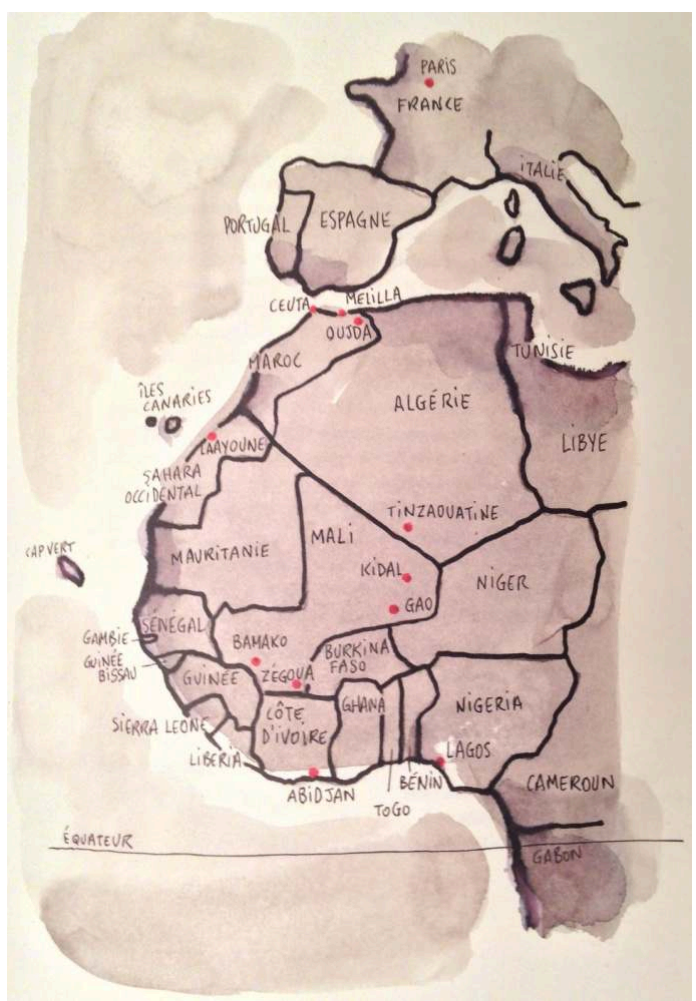

Extrait de : Bessora (auteur) ; Barroux (illustrateur) (2014) Alpha, Abidjan-Gare du Nord, Paris, Gallimard.

7 L'arrivée des bandes dessinées dans les rayons du fonds documentaire du laboratoire MIGRINTER confirme l'intérêt d'intégrer de nouveaux supports dans la compréhension des expériences migratoires. Considérés comme des outils d'expression par certains, comme des données de terrains de recherche à part entière par d'autres, la présence de ces œuvres réaffirme la pertinence de la diversification des sources d'analyse des flux migratoires dans le champ des migrations internationales.

\section{BIBLIOGRAPHIE}

Fernandez, Jacques (auteur) ; Camus, Albert (auteur adapté) (2013) L'étranger, Paris, Gallimard, 134 p. (Fétiche).

Boudjellal, Farid (1997) Le Beurgeois, Toulon, Soleil, 44 p. 
NOTES

1. cf. Diakhité, Bakary (2016) Sylvie Bredeloup, Migrations d'aventures. Terrains africains, e-Migrinter, $\mathrm{n}^{\circ} 14$.

\section{AUTEURS}

SARAH PRZYBYL

Doctorante en géographie, MIGRINTER - UMR 7301 CNRS / Université de Poitiers sarah.przybyl@univ-poitiers.fr 